\title{
Dark currents and their effect on the primary beam in an $X$-band linac
}

\author{
Karl L. F. Bane, Valery A. Dolgashev, Tor Raubenheimer, Gennady V. Stupakov, and Juhao Wu \\ Stanford Linear Accelerator Center, Stanford University, Stanford, California 94309, USA
}

(Received 26 April 2005; published 15 June 2005)

\begin{abstract}
We numerically study properties of primary dark currents in an $X$-band accelerating structure. For the H60VG3 structure considered for the Next Linear Collider (NLC) we first perform a fairly complete (with some approximations) calculation of dark-current trajectories. These results are used to study properties of the dark current leaving the structure. For example, at accelerating gradient of $65 \mathrm{MV} / \mathrm{m}$, considering two very different assumptions about dark-current emission around the irises, we find that the fraction of emitted current leaving the structure to be a consistent $\sim 1 \%$. Considering that $\sim 1 \mathrm{~mA}$ outgoing dark current is seen in measurement, this implies that $\sim 100 \mathrm{~mA}$ (or $10 \mathrm{pC}$ per period) is emitted within the structure itself. Using the formalism of the Liénard-Wiechert potentials, we then perform a systematic calculation of the transverse kick of dark currents on a primary linac bunch. The result is $\sim 1 \mathrm{~V}$ kick per $\mathrm{mA}$ (or per $0.1 \mathrm{pC}$ per period) dark current emitted from an iris. For an entire structure we estimate the total kick on a primary bunch to be $\sim 15 \mathrm{~V}$. For the NLC linac this translates to a ratio of (final) vertical beam offset to beam size of about 0.2. However, with the assumptions that needed to be madeparticularly the number of emitters and their distribution within a structure - the accuracy of this result may be limited to the order of magnitude.
\end{abstract}

DOI: 10.1103/PhysRevSTAB.8.064401

PACS numbers: 41.85.Ja, 41.20.-q, 41.75.Fr

\section{INTRODUCTION}

In high gradient, normal conducting accelerator structures electrons are emitted spontaneously from the walls and then move under the influence of rf fields. Of this "dark current," what leaves the structure is typically measured to be on the mA (average current) level. A question of interest for accelerators of low emittance beams is, What is the effect of dark-current electrons on bunches, e.g., can they significantly affect the orbit or emittance? This question has significant relevance for normal conducting linear colliders such as the Next Linear Collider (NLC/GLC) or the CLIC designs [1]. It has been suggested that dark currents might have been a source of beam jitter in the Stanford Linear Collider (SLC) [2]. In this report we study the dark current of an $X$-band structure and its impact on the beam in the NLC linac using simulations.

Dark currents have been studied by many authors, both experimentally (see, e.g., Refs. [3-5]) and through numerical tracking (see, e.g., Refs. [6-11]). A difference from earlier numerical work is that, instead of randomly choosing many times and positions within a structure for darkcurrent emission, that is then followed, we begin with a fairly complete calculation of possible emission times and positions (with some approximations). We then apply assumptions about the spacial distribution of emitters to weight these results. For this study, we use a tracking program, written in MATHEMATICA, by Setzer [8]. The program was originally written for standing wave structures; we have modified it to be applicable to traveling wave structures and to include the calculation of darkcurrent kicks on a primary beam moving through the structure. For purposes of normalization we will refer to measurements performed on the same $X$-band structure at SLAC, at the NLC Test Accelerator (NLCTA) [12].

In the first part of this report, we address such questions as: what fraction of dark current reaches the ends of a structure, what are the temporal and spectral distributions of outgoing dark current, and what is the dependence on accelerator gradient. Where possible these results are compared against measurements. In the second part of the report we use the Liénard-Wiechert potentials formalism to estimate the kick of the dark current on a primary beam traversing the structure. Note that this is a study of the behavior and effects of primary dark-current electrons in $X$-band accelerator structures and does not include effects of secondary electrons.

\section{SIMULATION PROGRAM}

Our simulation program can be applied to cylindrically symmetric, periodic, traveling wave structures of any length. First MAFIA [13] is used to obtain electric and magnetic fields (complex quantities) over a fine grid that covers one cell. This data is splined to give the fields as functions of radial and longitudinal coordinates $(r, z)$. The Floquet condition then gives the fields in any cell of a repeating structure. Finally, time dependence is added by multiplying (with the proper phase) with $e^{i \omega t}$, where $\omega$ is the (rf) radial frequency and $t$ is time.

The specific structure that we consider in this report is the H60VG3 disk-loaded structure, a 54-cell, approximately constant gradient cavity that was built for the NLC project and that operates at $11.4 \mathrm{GHz}$ at a per cell phase advance of $5 \pi / 6$ [14]. Note that our program approximates the fields of a constant gradient structure, but it does not include the gradual change in cavity dimension 


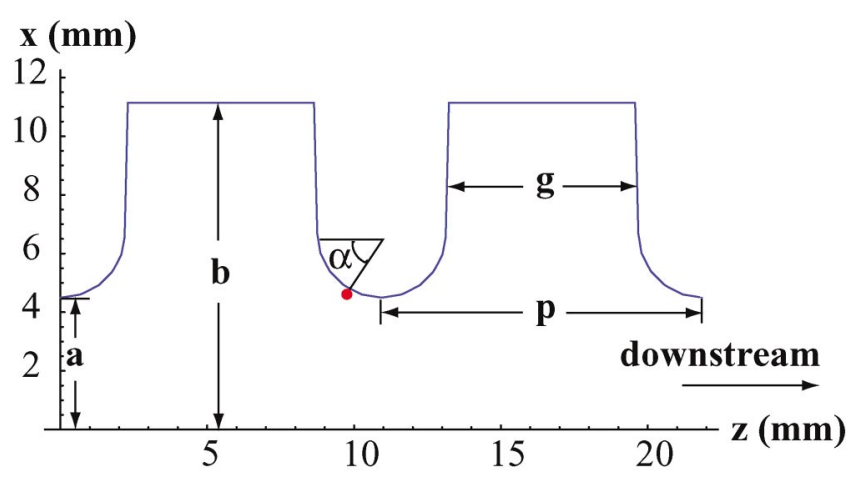

FIG. 1. (Color) Two cells of the model geometry, showing the angle $\alpha$ (the emission point is in the $x-z$ plane and indicated by the red dot).

found in the real structure. For our calculations we take a (cylindrically symmetric) lossless model with dimensions of the average H60VG3 cell: iris radius $a=4.7 \mathrm{~mm}$, cavity radius $b=11.1 \mathrm{~mm}$, gap $g=6.9 \mathrm{~mm}$, and period $p=10.9 \mathrm{~mm}$ (see Fig. 1).
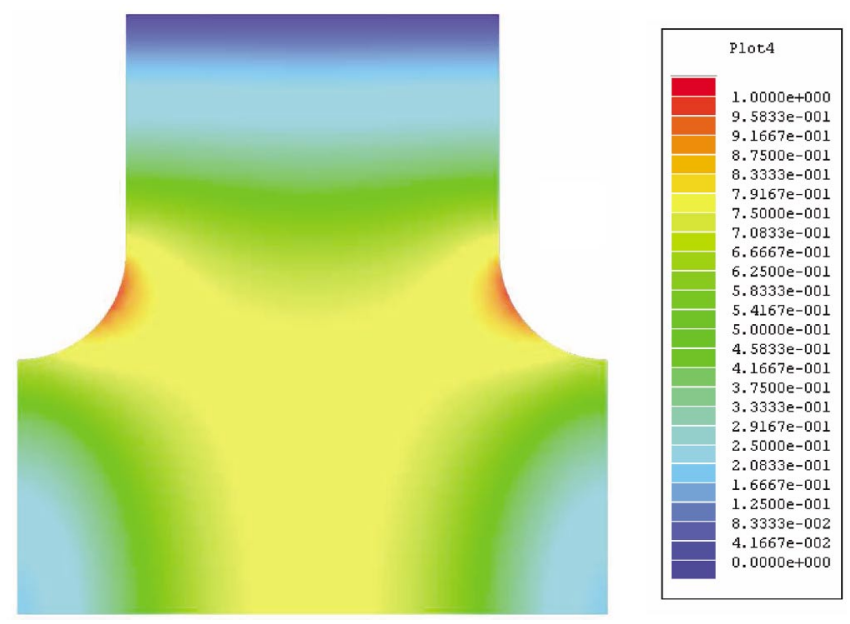

FIG. 2. (Color) Absolute value of the electric field in one cell of the H60VG3 structure.
In experiments at SLAC, at the NLCTA, a full cavity is connected at both ends to $20 \mathrm{~cm}$ of vacuum chamber and then to detectors (beam current monitors). In our simulations, in order to be able to compare with measurement the current reaching the detectors, we also add $20 \mathrm{~cm}$ tubes of radius $a$ (with no fields) to the ends of the structure. The correct end effects, including those due to the 3D nature of the coupling cells, are not included. Note that in our simulations, unless otherwise indicated, we scale the fields so that the accelerating gradient, when averaged over a cell, is $E_{\text {acc }}=65 \mathrm{MV} / \mathrm{m}$. The pattern of the absolute value of the electric field in one cell is shown in Fig. 2. In Fig. 3 we show the longitudinal electric field in the frame of a test particle moving through the structure on the crest of the rf wave (a), and by one moving (at the speed of light) against the wave (b). In Fig. 3(b) we see 5 oscillations over a superperiod of 6 cells, because the per cell phase advance of the mode is $5 \pi / 6$.

For dark-current simulations consider now emission in the $x-z$ plane, beginning on an iris at angle $\alpha$, defined in Fig. 1. In our figures of the structure, here and in what follows, emission is from above (positive $x$ ), and downstream, the direction of acceleration and power flow, is to the right. We allow charged macroparticles, initially at rest, to be pulled away from the walls by the rf fields. We let the time development of the charge of emitted particles follow the Fowler-Nordheim (FN) equation [15]

$$
J=1.54 \times 10^{-6} \frac{\beta^{2} E(t)^{2}}{\varphi} 10^{4.52 \varphi^{-0.5}} \exp \left[-\frac{6.53 \times 10^{9} \varphi^{1.5}}{\beta E(t)}\right]
$$

with $J$ the current density (in $\mathrm{A} / \mathrm{m}^{2}$ ), $\beta$ the field enhancement factor, $\varphi$ the work function of the metal (in eV), and $E(t)$ the applied surface electric field (in $\mathrm{V} / \mathrm{m}$ ). Note that, in our structure, the maximum of the surface field, $\hat{E}$, varies from 85-130 MV/m, depending on the location on an iris of the emission point. In our simulations we take $\varphi=$ $4.7 \mathrm{eV}$ (copper) and $\beta=30$, a value that has typically been found in measurement at the NLCTA [12]. Note

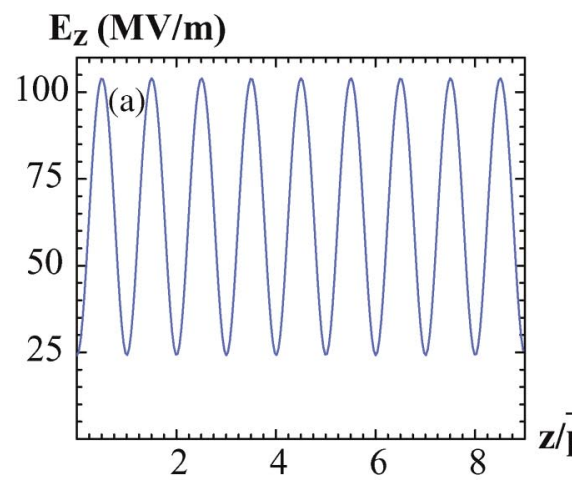

\section{$\mathbf{E}_{\mathbf{Z}}(\mathbf{M V} / \mathbf{m})$}

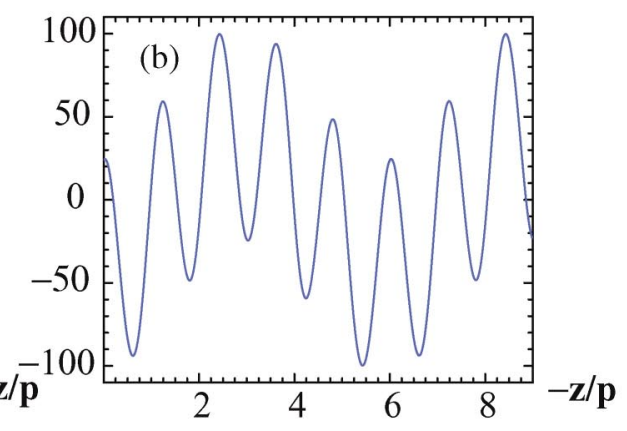

FIG. 3. (Color) The longitudinal electric field seen by a test particle moving with the rf wave on crest (a), and by one moving (at the speed of light) against the wave (b). 
that, as a function of time (or $\mathrm{rf}$ phase $\phi$ ) the emitted current is approximately Gaussian, with rms width $\sigma_{\phi}=$ $\sqrt{\beta \hat{E} /\left(6.53 \times 10^{9} \varphi^{1.5}\right)}$, which in our case varies from $11^{\circ}-14^{\circ}$. In our simulations we consider relative currents, and the cross-sectional area of emitters is not important (absolute current, however, will be deduced at the end from comparison with experiments at the NLCTA).

After macroparticles leave the wall they move under the influence of the Lorentz force due to the rf fields; their motion is tracked, with the aid of Boris rotation (see, e.g., [16]), until they either hit a wall or leave the structure. Among the simplifications in our calculations are

(i) The particles are assumed to have no initial energy and to be emitted perpendicular to the (ideal) metal surface. In reality particles emitted from a metal have initial energy on the order of a few $\mathrm{eV}$ and initial angle that will deviate from the normal [10]. These assumptions affect the longer term trajectory of the particles.

(ii) Dark current is thought to emit from local microscopic protrusions on the metal surface, which enhance the local electric field and will tend to change the (normal) emission direction. Such microscopic perturbations and
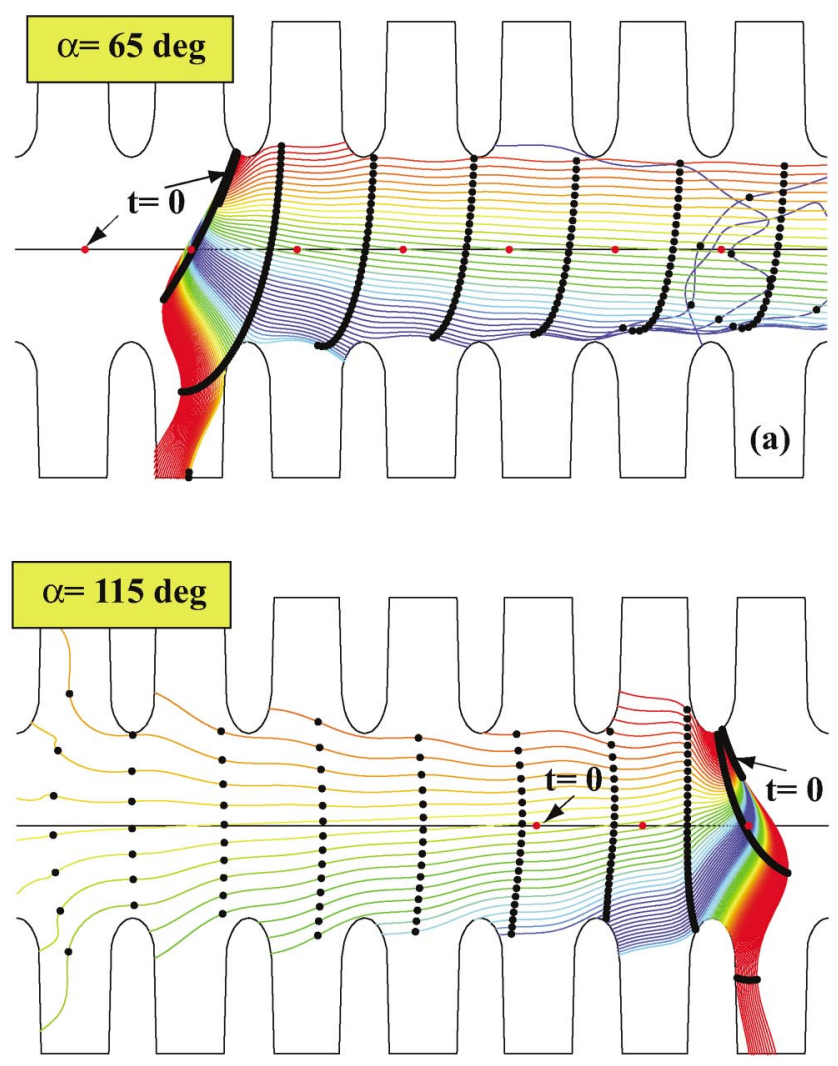

their effects on the beam dynamics are not included in our simulations.

(iii) The self-fields of the dark-current particles are assumed to be small and are not considered.

(iv) This is a study of primary dark-current electrons and their effects. In real structures, however, there will be secondaries also, and they may even outnumber the primary electrons (as was found, for example, in a high gradient S-band study [6]).

In Fig. 4 we plot the trajectories followed by darkcurrent macroparticles that are emitted at $\mathrm{rf}$ phase interval $\Delta \phi \equiv(180 / \pi) \omega \Delta t=1^{\circ}$, for 4 example iris emission locations (corresponding to $\alpha=65^{\circ}, 90^{\circ}, 115^{\circ}$, and $140^{\circ}$ ). Color coding gives relative charge of the macroparticle following the trace (maximum in each plot is blue, small is red), and also indicates relative time of emission. Only trajectories of particles with charge greater than $10^{-5}$ of the maximum value are shown. Black dots give snapshots of macroparticle positions, beginning with the time the last particle is emitted from the iris $(t=0)$, and then in time steps $c \Delta t=1 \mathrm{~cm}$ ( $c$ is speed of light). One on-axis $\mathrm{rf}$ crest position (for electrons) is also given at snapshot times, by red dots. (These will be referred to later when
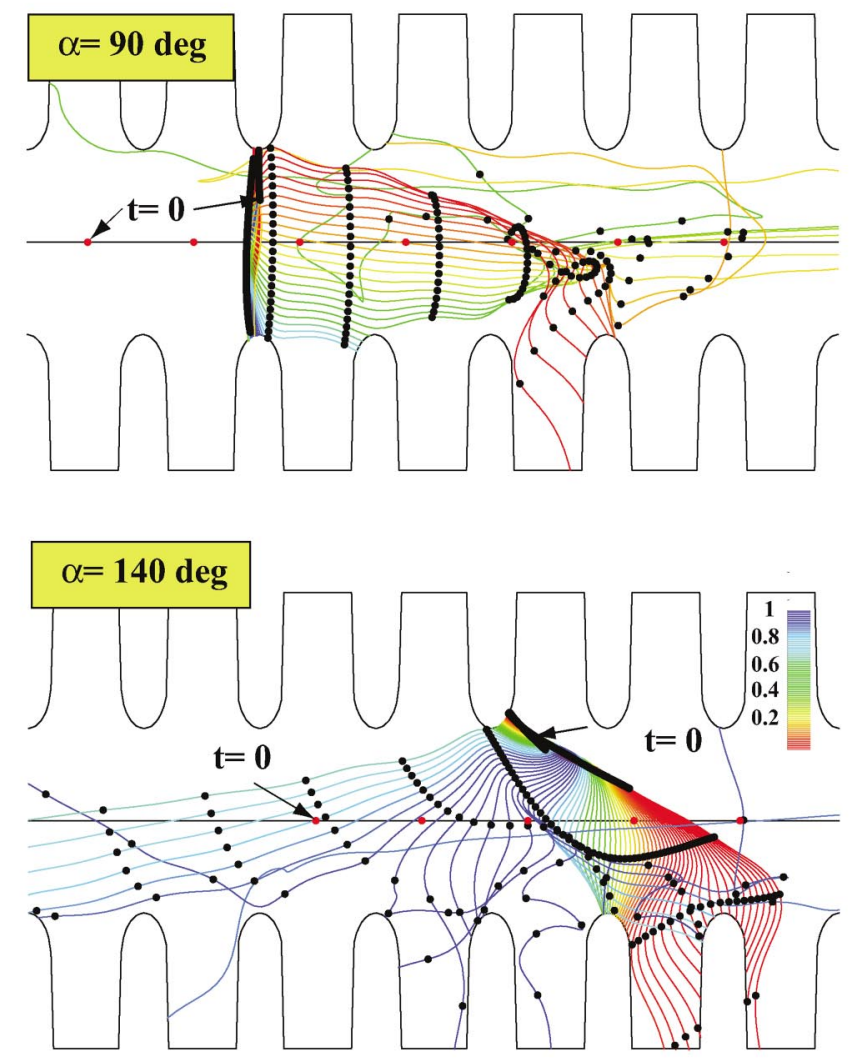

FIG. 4. (Color) Trajectories (in the $x-z$ plane) of dark-current macroparticles emitted at rf phase interval $\Delta \phi=1^{\circ}$, for 4 selected emission angles $\alpha$. Color coding indicates relative charge of the associated macroparticle. In each frame particles are emitted from an iris from above (positive $x$ ); downstream is to the right (positive $z$ ). Black dots give snapshots of the macroparticle positions, beginning with the time they have all left the iris surface $(t=0)$, and then in time steps $c \Delta t=1 \mathrm{~cm}$. One on-axis rf crest position is also shown at snapshot times (the red dots). 
we discuss the kicks of dark currents on the primary beam.)

For $\alpha$ near $0^{\circ}$ and $180^{\circ}$ (not shown) the dark current does not cross the axis and is confined to the cell neighboring the emitting iris. However, away from these regions we find that typically a large part of the dark current ends up colliding with the emitting iris (or neighboring cells) on the opposite side of the axis from the emission point. In Fig. 4 we see that the case $\alpha=65^{\circ}$ has significant "capture," by which we mean particles that become caught by the rf wave and travel long distances through the structure. We see that the captured particles are emitted later in time than peak emission. The captured dark current begins by filling almost the entire aperture of the cavity; gradually after many cells (not shown), due to adiabatic damping, the transverse beam size reduces. We note that the traces seem to vary rather uniformly from one to the next; however, there are a few (blue) traces that, after beginning like their neighbors, suddenly move in very different directions. From the black dots in the figure we see that, after initially moving upstream, the captured particles continue downstream coherently. The case $\alpha=115^{\circ}$, in contrast, shows significant upstream drift. The drifting particles are emitted earlier in time than peak emission, and the drift distance is limited to a half dozen or so cells.

As a practical device we now take a 108-cell, periodic model, have it emitting from the middle iris and calculate possible dark-current traces (with some resolution). We consider emission angles $\alpha$ from 0 to $180^{\circ}$ in $2.5^{\circ}$ steps, and all $\mathrm{rf}$ phases of emission, in fine steps of $\Delta \phi=0.1^{\circ}$. We store the information as large lists with elements $\{x, z$, $\left.p_{x}, p_{z}, t, \phi\right\}$ (position, momentum, time, and phase of the macroparticles) in time steps $c \Delta t \approx 1 \mathrm{~mm}$; the head of the list also includes the surface electric field at emission. Once these lists have been generated tracking no longer needs to be done; the lists themselves - with the aid of interpolation-suffice for the study of dark currents for any emission scenario in a 54-cell structure. These lists will be used in outgoing current and kicks to the primary beam studies that follow.

\section{OUTGOING CURRENT}

Let us study the outgoing current (both up- and downstream) from a 54-cell structure. We assume that there are many emitters, so that we can average over trajectories. From the stored lists of trajectories, by making assumptions about the $\alpha$ dependence of emitted current and performing sums, we obtain properties of the outgoing current in a 54-cell cavity.

First, to study capture, we calculate the fraction of emitted current that reaches 54 cells downstream, $I_{d n} / I_{e}$ ( $I_{e}$ is emitted current), as function of $\alpha$, in $2.5^{\circ}$ steps (see Fig. 5). We note that the curve is not smooth, due to the nonuniform nature of some trajectories; and that capture is

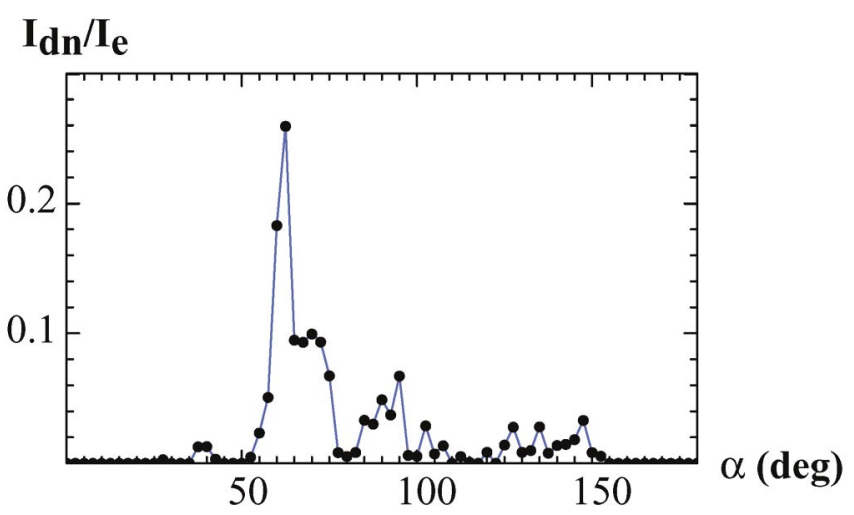

FIG. 5. (Color) Fraction of emitted current that reaches 54 cells downstream of emitting iris vs $\alpha$.

very sensitive to $\alpha$ and is maximized when emission is from the upstream end of an iris, at angle $62.5^{\circ}$.

Images of dark currents are routinely obtained during commissioning of rf guns; these images indicate that darkcurrent emitters tend to be relatively few and steady in time (see, e.g., [17] for $s$ band, [18] for $x$ band). Therefore, in our structure we expect there to be relatively few emitters on any iris. However, the $\alpha$ dependence of dark current emitted from an iris is not known. We will, therefore, make two very different assumptions about the $\alpha$ dependence and compare results, with the idea of obtaining a range in which the real answer is likely to lie. The first assumption, which we call the nonuniform emission assumption, is that the FN equation, with the same $\beta$, also applies at different iris positions. The maximum electric field on the iris surface varies from $85 \mathrm{MV} / \mathrm{m}$ at $\alpha=90^{\circ}$, to $130 \mathrm{MV} / \mathrm{m}$ at $\alpha=25^{\circ}, 155^{\circ}$ (see Fig. 2). Under the nonuniform emission assumption the importance of emission angles near $25^{\circ}, 155^{\circ}$, will be strongly enhanced. However, this assumption does not seem to agree with observation; e.g., pitting on iris surfaces, which is thought to be indicative of dark-current emission [19], is not preferentially found at the angles of maximum field. The second assumption about the $\alpha$ dependence, which we call the uniform emission assumption, is that the peak current density is independent of $\alpha$. A motivation for this assumption is to consider the FN equation to be applicable microscopically - with $\beta$ a measure of the spikiness of local protrusion on the surface. If the probability of finding a relatively large spike is independent of $\alpha$, we might then expect the uniform emission assumption to be the more applicable.

Figure 6 gives, for the uniform emission assumption, the fraction of emitted current that exits the structure (solid) and reaches the detectors (dashes), as functions of cell of origination, $n_{\text {cell }}$. We see from the relatively flat region of the downstream curves that many particles are captured. The large difference between both pairs of curves at the ends indicates that many particles generated near the ends 


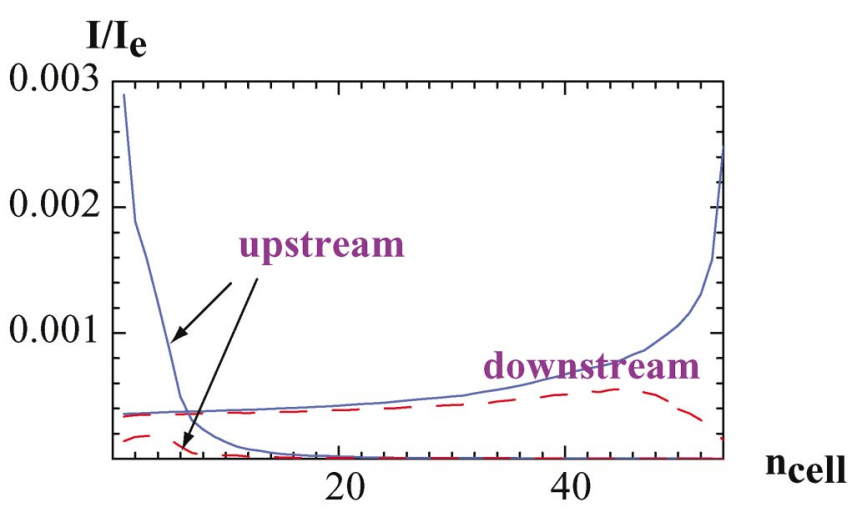

FIG. 6. (Color) For emission uniform with $\alpha$ : fraction of emitted dark current exiting the structure (solid) and reaching the detectors (dashes) as functions of cell of origination $n_{\text {cell }}$.

have large angles and are collimated away. The sum accumulated from the ends of the cavity gives the total current outgoing at the downstream and upstream ends; for the uniform (nonuniform) assumption 3.3\% (0.6\%) of emitted current leaves the downstream end of the structure; for the upstream end the results are $1.0 \%(0.6 \%)$. For the two very different emission assumptions, the results are of the same order. In the first two columns of Table I we summarize, for both uniform and nonuniform emission assumptions, the fraction of emitted dark current that leaves the structures, and also the fraction that makes it into the detectors.

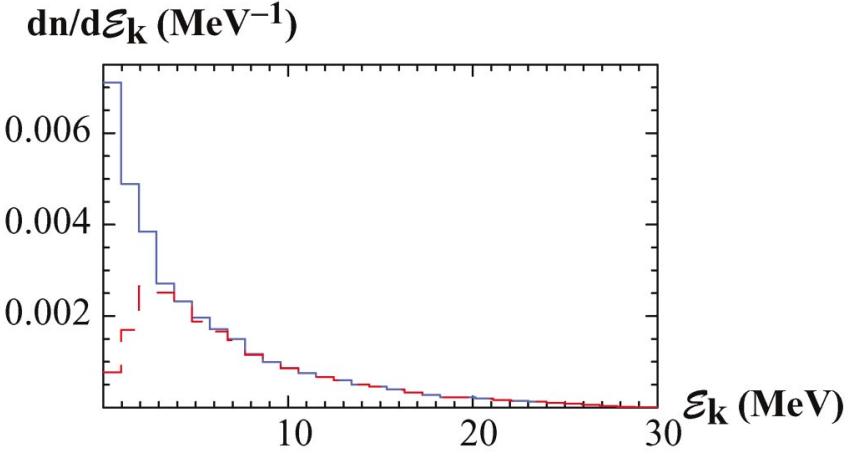

FIG. 8. (Color) For emission uniform with $\alpha$ : distribution in kinetic energy of dark current exiting the structure (solid) and entering the detectors (dashes), at the downstream end.

Figure 7 displays, for emission uniform in $\alpha$, the time distributions (normalized to emitted current) of the outgoing current at the ends of the structure and at the detectors (note that $\lambda$ is $\mathrm{rf}$ wavelength). We see that the downstream current is well bunched (for capture $0 \leq c t \leq$ $\lambda / 4$ ), whereas the upstream current is not. Note that the upstream current is mostly collimated away before reaching the detector. In Fig. 8 we display the distribution of the downstream kinetic energy $\mathcal{E}_{k}$, again for the uniform assumption. We see that it is low energy particles (which tend to have large angles) that are collimated away. Note that the maximum energy is $28 \mathrm{MeV}$, whereas $E_{\text {acc }} L=38 \mathrm{MV}$

TABLE I. Fraction of emitted dark current leaving ends of the structure, and reaching the detectors (first 2 columns); the $11.4 \mathrm{GHz}$ component of the absolute value of the Fourier integral of the exiting currents (in arbitrary units, last 2 columns).

\begin{tabular}{lcccc}
\hline \hline Emission dependence on $\alpha$ & $I_{d n} / I_{e}[\%]$ & $I_{u p} / I_{e}[\%]$ & FI-down & FI-up \\
\hline Uniform & 3.3 & 1.0 & 100 & 10 \\
Into detectors & 2.2 & 0.1 & 75 & 1.5 \\
Nonuniform & 0.6 & 0.6 & 17 & 3.5 \\
Into detectors & 0.3 & 0.05 & 10 & 0.5 \\
\hline \hline
\end{tabular}
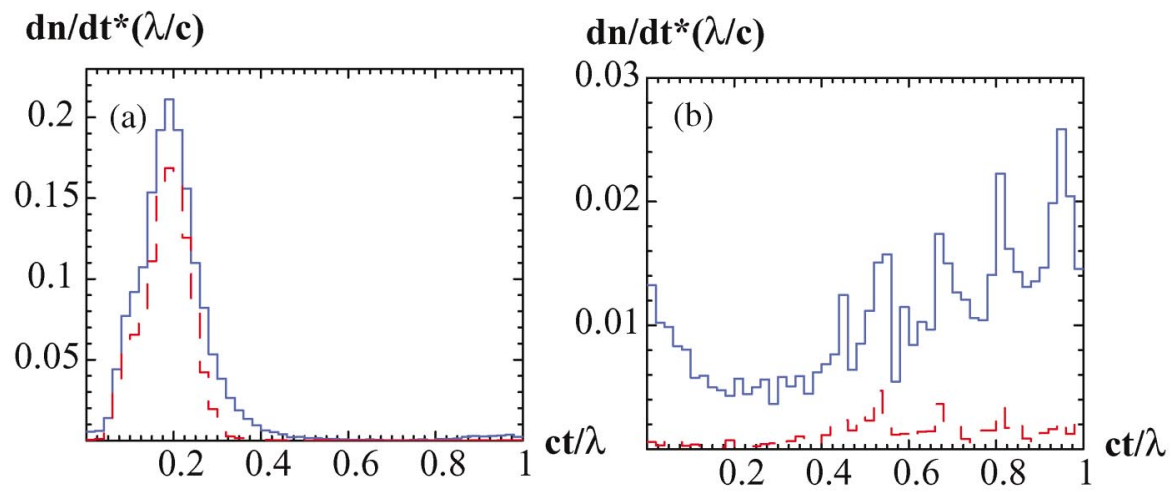

FIG. 7. (Color) For emission uniform with $\alpha$ : time distribution of dark current exiting the structure (solid) and entering the detectors (dashes), at the downstream (a) and upstream (b) ends. 
( $L$ is structure length). The upstream distributions (not shown) are rather uniform and limited to $\mathcal{E}_{k}<2 \mathrm{MeV}$.

The real detectors are cavities that are tuned to the structure frequency, $11.424 \mathrm{GHz}$, and the typical measured ratio of upstream to downstream current is about a factor of 10 [12]. To compare with measurement we compute the absolute value of the $11.424 \mathrm{GHz}$ component of the Fourier integral of the up and down time distributions (see the right two columns of Table I). We see that the ratio of the downstream to upstream Fourier components, after collimation, is 50 (20) for the uniform (nonuniform) emission assumption. Given the unbunched nature of the upstream outgoing current, such a rough agreement with measurement may be the best that we can hope for.

According to measurement $\sim 1 \mathrm{~mA}$ of current, averaged over an rf period, leaves the downstream end of the structure. Therefore, our calculations imply that $\sim 100 \mathrm{~mA}$ of average current (or a total of $\sim 10 \mathrm{pC}$ of charge over one period) is emitted within the structure itself.

\section{A. Accelerator gradient dependence}

We have repeated the simulations for different gradients. In Fig. 9 we give the gradient dependence of the fraction of emitted current reaching the downstream (a), and upstream (b) detectors, when assuming uniform (solid line) and nonuniform (dashes) emission. We note that capture rises steeply near an (on-axis) acceleration of $E_{\text {acc }}=$ $50 \mathrm{MV} / \mathrm{m}$. Note that this is less than the gradient necessary to capture a particle from rest, $E_{\text {acc }}=\pi m_{0} c^{2} / \lambda=$ $61 \mathrm{MV} / \mathrm{m}$ [20]. Finally, in Fig. 10 we plot the downstream data as $\ln \left(I_{d n} / E_{\text {acc }}^{2.5}\right)$ vs $1 / E_{\text {acc }}\left(I_{d n}\right.$ is outgoing dark current, in arbitrary units). Measured dark current is often plotted this way, fitted to a straight line, and the slope is used to obtain the $\beta$ to characterize a structure [19]. Our fitted slopes are $13 \%$ steeper than the emitted current curve. Such a measurement procedure will, therefore, overestimate the effective $\beta$ within a structure by $13 \%$. Nevertheless, for outgoing current, we see that the FN

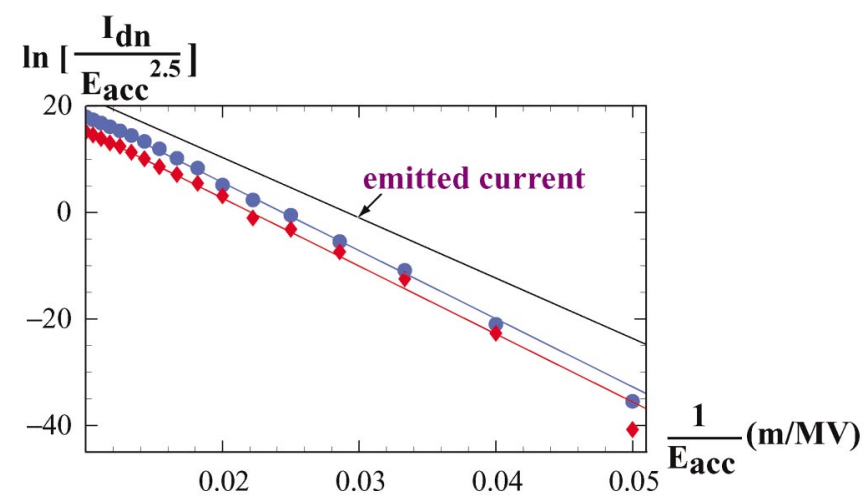

FIG. 10. (Color) Fowler-Nordheim plot of dark current reaching downstream detector, assuming uniform (circles) or nonuniform (diamonds) emission. Lines give linear fits and the emitted current.

dependence (of emission) completely overwhelms such a "capture" dependence.

\section{LIÉNARD-WIECHERT POTENTIALS}

We next calculate the effect of dark current on a primary beam (a bunch) as it moves on-axis at the speed of light through the structure. (Selected primary bunch properties in the NLC linac are given in Table II.) In the NLC the (electron) bunch rides within $20^{\circ}$ of rf crest (it is varied from the crest to compensate wakefield effects), while the

TABLE II. Selected bunch properties in the NLC linac.

\begin{tabular}{lcc}
\hline \hline \multicolumn{1}{c}{ Parameter } & Value & Units \\
\hline Charge, $\mathrm{Q}$ & 1 & $\mathrm{nC}$ \\
Rms bunch length, $\sigma_{z}$ & 100 & $\mu \mathrm{m}$ \\
Energy, $\mathcal{E}$ & $10-250$ & $\mathrm{GeV}$ \\
Vertical rms size, $\sigma_{y}$ & $4-1$ & $\mu \mathrm{m}$ \\
Horizontal rms size, $\sigma_{x}$ & $40-10$ & $\mu \mathrm{m}$ \\
\hline \hline
\end{tabular}
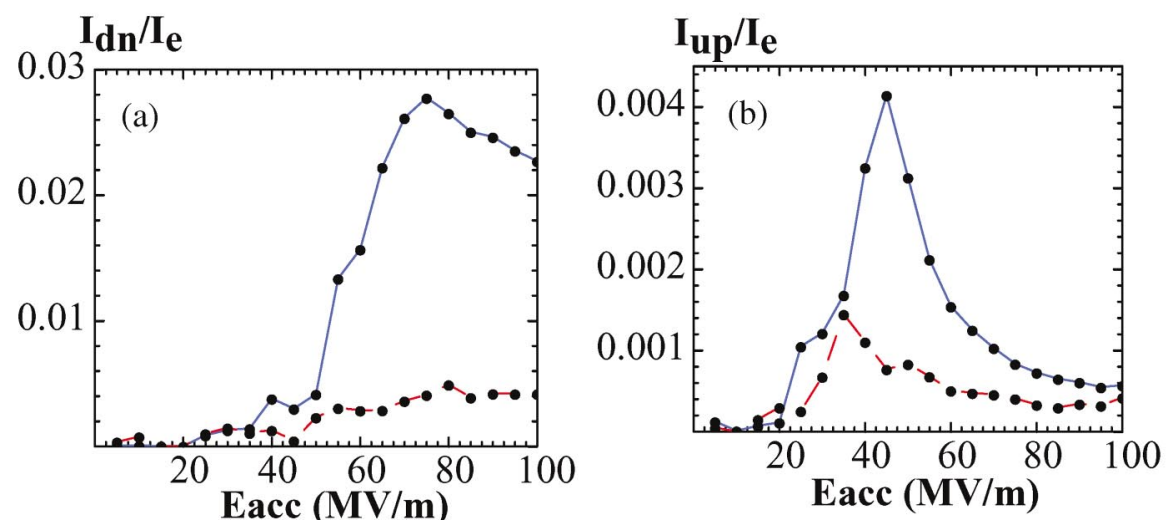

FIG. 9. (Color) Gradient dependence of fraction of emitted current reaching downstream (a) and upstream (b) detectors, for uniform (solid line), nonuniform (dashes) emission. 
positron bunch is shifted by half a wave length. In the following we present detailed calculations for an electron test particle on crest only. At the end we discuss also the result for other rf phases. Finally, we take the scale of these results to estimate the dark-current kick on the NLC primary beam.

We can visualize, back in Fig. 4, the progression of the kicks on the test particle as it moves through the structure. Recall that the black dots give snapshots of the darkcurrent particles, beginning at the time the last particle is emitted from the iris $(t=0)$, and then in time steps of $c t=$ $1 \mathrm{~cm}$. One on-axis rf crest position (for electrons) is also shown at the snapshot times, by the red dots. The specific crest position that is shown is the one for which a comoving test particle would experience the largest kick from the emitted dark current. Consider, for example, the case $\alpha=$ $65^{\circ}$ [Fig. 4(a)]. We see that in this case such a test particle collides with dark-current particles of relatively high charge (blue in the figure) at time $c t \approx 1 \mathrm{~cm}$; after collision the test particle overtakes the dark-current particle, and then moves ever further ahead of it down the structure. From the 4 frames of the figure it seems that the largest dark-current-beam interaction (the collision) tends to be confined to the vicinity (in $z$ ) of the emitting iris or the neighboring cells. Thus we expect the effect to be essentially the same, no matter from which iris (of a multicell cavity) emission occurred.

We approximate the kick on a test particle (of the primary beam) due to a source particle (of the dark current) using the Liénard-Wiechert potentials between two particles moving in free space. This will not satisfy the boundary conditions at the cavity walls. But since the test particle never comes close to the walls, and since the dominant contribution to the kick it experiences comes when source particles come in close approach, this approximation should suffice. The electric and magnetic fields due to the Liénard-Wiechert potentials between source and test particles are [21]

$$
\begin{aligned}
\mathbf{E}(\mathbf{x}, t)= & q\left[\frac{\mathbf{n}-\boldsymbol{\beta}}{\gamma^{2}(1-\boldsymbol{\beta} \cdot \mathbf{n})^{3} R^{2}}\right]_{\mathrm{ret}} \\
& +\frac{q}{c}\left[\frac{\mathbf{n} \times\left\{(\mathbf{n}-\boldsymbol{\beta}) \times \dot{\boldsymbol{\beta}}_{\}}\right.}{(1-\boldsymbol{\beta} \cdot \mathbf{n})^{3} R}\right]_{\mathrm{ret}} \\
\mathbf{B}(\mathbf{x}, t)= & {[\mathbf{n} \times \mathbf{E}]_{\mathrm{ret}}, }
\end{aligned}
$$

with $\mathbf{x}$ test particle position and $t$ time; where $q$ is charge and $\boldsymbol{\beta} \equiv \mathbf{v} / c$ with $\mathbf{v}$ the velocity of the source particle and $\gamma$ its Lorentz energy factor. The quantities on the right hand side of Eqs. (2) are evaluated at the retarded time $t_{r}$, defined as the solution to

$$
c\left(t-t_{r}\right)=\left|\mathbf{x}(t)-\mathbf{x}_{s}\left(t_{r}\right)\right| \equiv R,
$$

where $\mathbf{x}_{s}$ designates the source position. The parameter $\mathbf{n}$ is the unit vector in the direction $\mathbf{x}(t)-\mathbf{x}_{s}\left(t_{r}\right)$; dot over a symbol indicates differentiation with respect to time. The first term in Eq. (2) is called the "velocity field" (or "space charge field") and the second term the "acceleration field."

The net kick on a test particle due to a dark-current particle is obtained by inserting these fields in the Lorentz force equation and integrating over time. The charge $q$ is normalized to total charge emitted per rf period, $q_{e}=$ $I_{e} \lambda / c,\left(I_{e}\right.$ is current emitted per period); as before, we normalize in this way so we do not need to know details of the emitter. Then, the total kick $k_{\text {tot }}$ is obtained by summing over the kicks from all the macroparticles $k_{n}: k_{\mathrm{tot}}=\sum_{n} k_{n}$. And finally, as before, we obtain a kick in absolute units with the help of NLCTA experience. Note that the essentially local (to the emitting iris) nature of the kick means that the result of one emission in a multicell structure can be taken to be independent of which iris actually emits; this fact will simplify our later calculation of the effect in a structure, where we assume that many irises emit.

\section{A. Kick divergence}

Consider now dark current that is emitted in the $x-z$ plane, and a test particle (of a primary beam) that is slightly offset in $y$. The force experienced by the test particle due to a close encounter with a dark-current macroparticle scales as $\sim 1 / d^{2}$, where $d$ is the distance between the two particles. For a continuous string of dark current it scales as $\sim 1 / d$; and if we integrate over time to obtain the total kick experienced by the test particle, we are left with a $\sim \ln d$ dependence, which for small $d$ is a weak divergence. (Note that the divergence is only in $y$, since in $x$ the kicks are antisymmetric about the collision point, as discussed below.) In a real situation, however, there are factors that will always cause us to have a finite kick. These include, for example, the fact that the dark current has a finite emittance; that the emitted trajectory will tend to have a slight azimuthal dependence (due to, e.g., surface irregularities) and will miss the structure axis (and thus the primary beam); that the primary beam itself (with its small transverse dimensions) will tend to be slightly off axis in $y$ and be missed by the dark-current beam; and that dark-current particles approaching closely will be kicked away by the field of the primary beam.

However, the main reason for dark-current particles to miss the test particle may be due to (microscopic) surface irregularities. Suppose a local protrusion of size $\sim 1 \mu \mathrm{m}$ emits a dark-current particle. If the electric field at the surface is $100 \mathrm{MV} / \mathrm{m}$, the particle will have gained $100 \mathrm{eV}$ in $1 \mu \mathrm{m}$ of travel, of which, let us suppose, $10 \mathrm{eV}$ is in $y$ (perpendicular to the nominal emission plane). By the time of collision (with the test particle) the dark-current particle will have drifted in $y$ by $\Delta y \approx$ $\beta_{y 0} s \sqrt{(1+\delta)^{2}-1} / \delta$. Here $\beta_{y 0}=v_{y 0} / c$ the initial (vertical) particle velocity and $s$ is the distance traveled by the particle before collision; $\delta=e E s / m_{0} c^{2}$, with $E$ the typi- 
cal electric field in the direction of motion and $m_{0}$ the rest mass of the electron.

For an initial $10 \mathrm{eV}$ vertical component of energy, taking $s=1 \mathrm{~cm}$ and $E=65 \mathrm{MV} / \mathrm{m}$, we find that by the time of collision (with the test particle) the dark-current particle will have drifted $\sim 100 \mu \mathrm{m}$ in the $y$ direction. Considering that, in the NLC linac, the vertical rms beam size varies from $4 \mu \mathrm{m}$ in the beginning to $1 \mu \mathrm{m}$ at the end (see Table II), we see that the chance of collision between dark current and test particles is small. In following calculations we offset the test particle trajectory by $100 \mu \mathrm{m}$ in $y$, perpendicular to the $(x-z)$ emission plane. The results are not sensitive to the exact amount (as will be seen when we present the results).

An approximation in our calculations that also results in a finite kick is that the dark-current particles are unaffected by the fields of the primary beam, whereas in reality particles that come close in approach will tend to be deflected away (the validity of ignoring this "recoil" will be explored later).

\section{B. Calculations}

To illustrate the calculations we will give, in the next 3 figures, plots of intermediate results for the case of test particle on crest, and dark-current emission angle $\alpha=65^{\circ}$ and emission phase interval $\Delta \phi=1^{\circ}$ [the traces shown in Fig. 4(a)]. First, the retarded time solutions for this example [the solutions to Eq. (3)] are shown in Fig. 11. We see that one of the blue traces has the closest approach to the $t_{r}=t$ line, due to a near collision between dark current and test particles; after the test particle passes the darkcurrent particle, the retarded time $t_{r}$ for this trace remains fixed at the time of collision.

Next the Liénard-Wiechert Equations, Eqs. (2), need to be solved for the $E$ and $B$ fields experienced by the test particle, as functions of time. Note that by the time of near collision between the dark current and test particles, the dark-current energy is still relatively low and the space

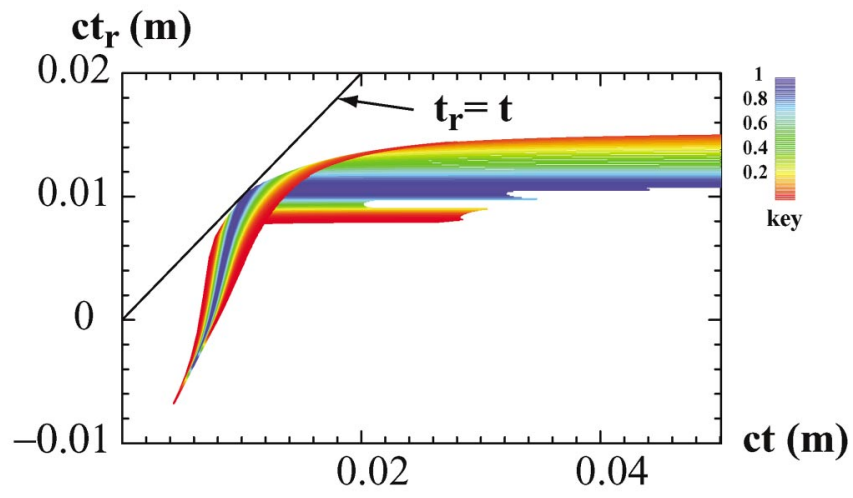

FIG. 11. (Color) Retarded time $t_{r}$ as function of time $t$ for the example trajectories of Fig. 4(a). Color code indicates relative macroparticle charge. charge component, with its $1 / \gamma^{2}$ factor, tends to dominate over the acceleration component; in addition, the $E$ force dominates over the $B$ force. In Fig. 12 we plot the space charge component (subscript $s$ ) of the electric field in $x$ and $y$ as function of time $t$ for the different dark-current macroparticles shown in Fig. 4(a). High charge (blue) traces give the largest contribution (on this scale the curves with color other than blue are all near zero). The maximum field is at time $c t \approx 1 \mathrm{~cm}$, as we could already see from Fig. 4(a). In the $x$ direction, earlier emitted macroparticles cross the axis before the test particle arrives, and therefore give a kick back, in the positive $x$ direction; and the opposite is true for macroparticles emitted later in time. Note that there are fields in the $z$ direction also, that are roughly antisymmetric about the collision point; the kicks, however, are weak, are of not much practical interest for a linear collider, and will not be discussed further here.

Integrating the Lorentz force due to the fields over time, we obtain the total kick due to each macroparticle, $k_{n}$ (see Fig. 13). We clearly see that the space charge component of the kicks dominates, and the $E$ kicks dominate over the $B$ kicks. Also, in $x$ we have large cancellation in the kicks due to the different macroparticles. Summing over all macroparticle contributions we obtain the total kick $k_{\text {tot }}$ for given emission angle $\alpha$. To obtain an accurate result we have repeated the entire process, but with the much finer macroparticle emission spacing $\left(\Delta \phi=0.1^{\circ}\right)$, and for $\alpha$ in steps of $5^{\circ}$. The results are shown in Fig. 14.

We see two what look like resonances, near $\alpha=60^{\circ}$ and $\alpha=125^{\circ}$, where the $y$ kick reaches a peak and the $x$ kick crosses zero. The former (latter) is a case where the macroparticles are moving downstream (upstream) at the time of collision. The general features of the curves are understandable: the charge of the macroparticles is symmetric about the phase at peak emission; at the "resonant" values of $k_{\text {tot }}$ the timing is such that a maximum charge macroparticles comes in closest approach with the test particle, which leads to a maximum kick in $y$ and (because of the symmetry in the charge) near cancellation of kick in $x$. Note that the transverse kicks are $\sim 1 \mathrm{~V}$ per $\mathrm{mA}$ average emitted current.

We have repeated the calculations with the test particle's offset in $y$ (in the out of plane direction) reduced from 100 to $25 \mu \mathrm{m}$; we find that $k_{\text {tot }}(\alpha)$ in $x$ is virtually unchanged, and in $y$ is changed by $\lesssim 30 \%$. We have also repeated the calculations using test particles that in $z$ are not on the $\mathrm{rf}$ crest (remember: in the NLC linac the electron beam can be shifted - for wakefield compensation - by up to $20^{\circ}$, and the positron beam is shifted by half a wavelength). The results are that the details of $k_{\text {tot }}(\alpha)$ are different, though the scale and maximum value are about the same.

Finally, to investigate the validity of ignoring the effect of the primary beam's fields on the motion of the dark current (the recoil of the dark current), we compare the kinetic energy in the dark current to the kick on the primary 


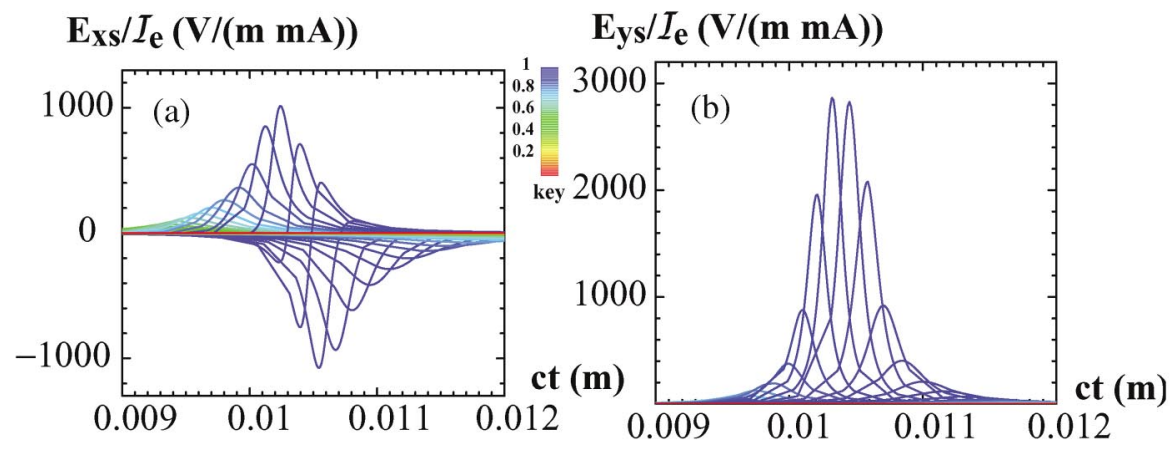

FIG. 12. (Color) Space charge part of electric field components $E_{x s}$ (a) and $E_{y s}$ (b) as functions of time $t$ as experienced by a test particle on the rf crest for the example of Fig. 4(a). The fields are normalized to average emitted dark current. Color code indicates relative macroparticle charge.
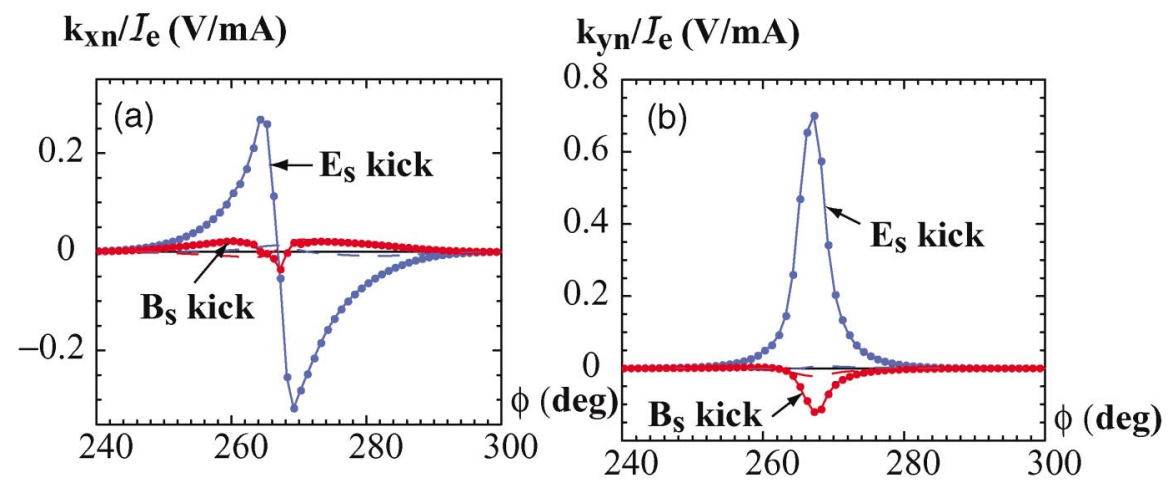

FIG. 13. (Color) Contribution of the individual (dark current) macroparticles to kicks in $x$ (a) and $y$ (b), experienced by a test particle (on crest) for the example of Fig. 4(a). The macroparticles are indexed by rf phase at emission, $\phi$ (their spacing is $1^{\circ}$ ). The $E(B)$ field contribution is shown in blue (red); the space charge (acceleration) fields contribution is indicated by solid (dashed) curves.

beam. Consider the quantity $k_{e} \equiv n_{k} \mathcal{E}_{k} \lambda /(e c Q)$, with $n_{k}$ the fraction of dark current that contributes most of the kick, $\mathcal{E}_{k}$ the kinetic energy of these particles at time of near approach, and $Q$ the charge of the primary beam $(=1 \mathrm{nC}$ for the NLC). We expect that our approximation (ignoring

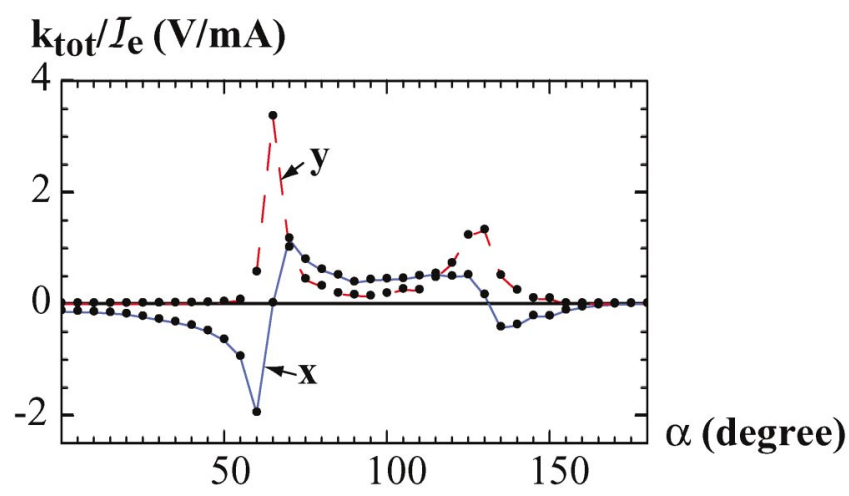

FIG. 14. (Color) Total kick in $x$ (solid) and $y$ (dashes) experienced by a test particle (on crest) as function of dark-current emission angle $\alpha$. For the calculation a fine macroparticle emission spacing was used $\left(\Delta \phi=0.1^{\circ}\right)$. the recoil in the kick calculations) is good provided that $k_{e}$ is large compared to our result of $1 \mathrm{~V} / \mathrm{mA}$, otherwise our result is an overestimate. For example, for the NLC with $\alpha=65^{\circ}$ we find that $n_{e}=0.2, \mathcal{E}_{k}=50 \mathrm{keV}$, and thus $k_{e}=0.9 \mathrm{~V} / \mathrm{mA}$; with $\alpha=130^{\circ}, \quad n_{e}=0.07, \quad \mathcal{E}_{k}=$ $150 \mathrm{keV}$, and $k_{e}=0.9 \mathrm{~V} / \mathrm{mA}$. It appears that for the NLC the dark-current recoil is important, and our result of $1 \mathrm{~V} / \mathrm{mA}$ kick should be taken as an overestimate.

\section{Kick on primary beam in the NLC linac}

We have found that the transverse kick due to dark current is on the order of $1 \mathrm{~V}$ per $\mathrm{mA}$ of average current (or per $0.1 \mathrm{pC}$ of charge per rf period) emitted from an iris. In the first half of this report we estimated that a total of about $100 \mathrm{~mA}$ average current is emitted within the structure. Now, assuming one emitter on each iris ( 54 emitters in all), each emitted at random azimuthal angle, we obtain a total kick on the primary beam of about $100 / \sqrt{54}=14 \mathrm{~V}$ per structure.

In the NLC main linac the average beta function varies with energy as $\bar{\beta} \sim \mathcal{E}^{1 / 2}$. In such a case the rms beam offset at the end of the linac due to all dark-current kicks, $\Delta y_{\mathrm{rms}}$, divided by the beam size, $\sigma_{y f}$, is given by (see, e.g., [22]) 


$$
\frac{\Delta y_{\mathrm{rms}}}{\sigma_{y f}}=\frac{e\left(k_{\mathrm{tot}}\right)_{\mathrm{st}}}{\mathcal{E}_{0}} \sqrt{\frac{N_{\mathrm{st}} \bar{\beta}_{y 0} \gamma_{f}}{2 \epsilon_{y n}}} f\left(\mathcal{E}_{f} / \mathcal{E}_{0}\right),
$$

with $f(x)=\sqrt{2\left(x^{1 / 2}-1\right) /(x[x-1])}$; with $\left(k_{\text {tot }}\right)_{\mathrm{st}}$ the kick from one structure, $N_{\text {st }}$ the number of structures, $\gamma$ the Lorentz energy factor, and $\epsilon_{y n}$ the normalized vertical emittance; with subscript $0(f)$ denoting an initial (final) quantity. For the NLC we take [1]: $\left(k_{\mathrm{tot}}\right)_{\mathrm{st}}=14 \mathrm{~V}, N_{\mathrm{st}}=$ $20000, \bar{\beta}_{y 0}=10 \mathrm{~m}, \epsilon_{y n}=3 \times 10^{-8} \mathrm{~m}, \mathcal{E}_{0}=10 \mathrm{GeV}$, and $\mathcal{E}_{f}=250 \mathrm{GeV}$; we find that $f\left(\mathcal{E}_{f} / \mathcal{E}_{0}\right)=0.12$ and $\Delta y_{\mathrm{rms}} / \sigma_{y f}=0.2$.

The many uncertainties in the calculation-e.g., the total emitted current within a structure, the number of emitters, and the position dependence of the emitted currents - means that our final result has maybe an order of magnitude accuracy. Nevertheless, the results indicate that the dark-current kicks are a small effect for the NLC. This is true provided that the dark-current distribution is relatively stable from pulse-to-pulse, as seems to be observed experimentally (see, e.g., $[5,17,18]$ ); then the transverse kicks would also be static or vary slowly in time and can be corrected with feedback. However, if it is not stable it could be a problem for the NLC, by significantly increasing the projected emittance of a train of bunches. Note that an important reason that the kick of the dark current is relatively small is that the total charge in the emitted dark current is small, only about $10 \mathrm{pC}$ per rf period per structure.

We have been talking about an effect on the vertical orbit of the primary beam. For an effect on the (single bunch) vertical emittance one needs a differential kick over a bunch, and the differential kick for the NLC bunch must be very small. For a noticeable effect a significant part of the emitted dark current would need to collide directly with the beam, but such a collision is very unlikely since the vertical beam size is very small compared to the typical dark-current offset. Thus we expect the effect of darkcurrent kicks on beam emittance to be negligibly small.

\section{CONCLUSION}

We have numerically studied properties of primary dark currents in an $X$-band accelerating structure. Unlike as is typically done in such calculations - starting with many random emission times and positions and then tracking the dark current - we begin with a fairly complete calculation of possible emissions (with some approximation) that we follow, and then apply assumptions about the spacial distribution of emitters to weight the earlier results.

For the H60VG3 structure considered for the Next Linear Collider (NLC) we give results on the outgoing dark current and its time and spectral properties (at accelerating gradient of $65 \mathrm{MV} / \mathrm{m}$ ) and the gradient dependence. For example, considering two very different assumptions about dark-current emission around the irises, we obtain the fraction of emitted current leaving the downstream end of a structure to be a consistent $\sim 1 \%$. Since, according to measurement, $\sim 1 \mathrm{~mA}$ of (average) dark current leaves the downstream end of a structure, this result implies that there is $\sim 100 \mathrm{~mA}$ of current (or $10 \mathrm{pC}$ total charge per rf period) emitted within a structure. In comparison with measurements using cavity monitors at the ends of a structure, where the ratio of downstream to upstream dark current is found to be about a factor of 10 , our result is between 20 and 50 .

The H60VG3 structure is meant to be used in the NLC linac, and we have studied also the kick of the dark current on a primary beam in such a linac. Using the formalism of the Liénard-Wiechert potentials we find the transverse kick to be $\sim 1 \mathrm{~V}$ per $\mathrm{mA}$ of average dark current (or per $0.1 \mathrm{pC}$ per rf period) emitted from an iris. (Since recoil of darkcurrent particles is not included in our calculations and does not appear to be insignificant, this result should be taken as an overestimate.) The expected kick on the primary beam, $\sim 15 \mathrm{~V}$ per structure, translates to a final ratio of (vertical) rms offset to beam size of 0.2. This result depends on many assumptions - such as the total emitted current within a structure and the number of emitters - and may be accurate in order of magnitude only. Meanwhile, the dark-current effect on (single bunch) vertical emittance should be negligibly small.

Given the apparent stability of dark current as seen in measurement, it seems that dark-current kicks will not be a significant effect in an $X$-band linear collider. Studies are still needed to determine whether this statement is still true for the higher gradients and smaller beam emittances of the CLIC design. Finally, we should note that this study also suggests that $\mathrm{rf}$ breakdown events may be a significant source of beam jitter in a collider because the emitted currents are thousands of times higher, and they vary in time and thus cannot be compensated with feedback [23].

\section{ACKNOWLEDGMENTS}

The authors thank S. Setzer for providing us with his computer program. We also thank R. Miller and M. Pivi for useful discussions. This work was supported by the Department of Energy, Contract No. DE-AC0276SF00515

[1] International Linear Collider Technical Review Committee, SLAC Report No. SLAC-R 606, 2003.

[2] F.-J. Decker et al., in Proceedings of Linac 1996 Conference, Geneva, Switzerland, edited by C. Hill and M. Vretenar (CERN, Geneva, Switzerland, 1996), p. 143.

[3] J. Wang and G. Loew, IEEE Trans. Nucl. Sci. 32, 2915 (1985).

[4] H. Matsumoto et al., in Proceedings of the Particle Accelerator Conference, San Francisco, California, 1991 (IEEE, Piscataway, NJ, 1991), p. 2061. 
[5] J. Norem et al., Phys. Rev. ST Accel. Beams 6, 072001 (2003).

[6] S. Yamaguchi, Report No. LAL/RT 92-18, Orsay, 1992.

[7] H. Padamsee et al., in Proceedings of the Particle Accelerator Conference, Washington, DC, 1993 (IEEE, Piscataway, NJ, 1993), p. 998.

[8] S. Setzer et al., in Proceedings of the Particle Accelerator Conference, Washington, DC, 1993 (Ref. [7]), p. 3566.

[9] V. Ivanov et al., in Proceedings of the Particle Accelerator Conference, Portland, Oregon, 2003 (IEEE, Piscataway, NJ, 2003), p. 2664.

[10] V. Balandin et al., TESLA Report No. 2003-10, DESY, 2003.

[11] V. A. Dolgashev et al., in Proceedings of the European Particle Accelerator Conference, Lucerne, Switzerland (CERN, Geneva, Switzerland, 2004), p. 2203.

[12] C. Adolphsen and S. Doebert (private communication).

[13] MAFIA Collaboration, User guide, CST-GmbH, Darmstadt, Germany.

[14] J. Wang et al., Report No. SLAC-PUB 10711, SLAC, 2004.
[15] R. Fowler and L. Nordheim, Proc. R. Soc. A 119, 173 (1928).

[16] R. Hocknew and J. Easterwood, Computer Simulation Using Particles (IOP Publishing Ltd., London, 1988).

[17] Xi-jie Wang, Ph.D. thesis, UCLA [Institution Report No. UMI92-21016, 1992].

[18] A. Vlieks (private communication).

[19] J. Wang, Ph.D. thesis, Stanford University [Institution Report No. SLAC-R-339, 1989].

[20] R. Helm and R. Miller, in Linear Accelerators, edited by P. Lapostolle and A. Septier (North-Holland, Amsterdam, 1970), pp. 115-146.

[21] J.D. Jackson, Classical Electrodynamics (John Wiley \& Sons, New York, NY, 1998), 3rd ed.

[22] K. Bane et al., in Proceedings of European Particle Accelerator Conference, London, England, 1994, edited by V. Suller and Ch. Petit-Jean-Genaz (World Scientific, River Edge, NJ, 1994), p. 1114.

[23] V.A. Dolgashev and T. Raubenheimer, SLAC Report No. SLAC-PUB 10668, 2004. 